\title{
Human Agency and Global Catastrophic Biorisks
}

$\mathrm{G}$ IVEN THat EVEnTS SUCH as The Black Death and the introduction of smallpox to the Americas have comprised some of the greatest catastrophes in human history, it is natural to examine the possibility of global catastrophic biological risks (GCBRs). As Schoch-Spana et al note, ${ }^{1}$ a defining characteristic of GCBRs is their intergenerational consequences. In the particularly extreme case of human extinction or permanent collapse of human civilization, such GCBRs would jeopardize the very existence of many thousands of future generations.

Does the category of GCBR merit special research effort? The probability of GCBRs, extinction level or otherwise, is presumably much lower than other types of biological risk. Moreover, as with many low-probability, high-consequence risks, the uncertainties involved are massive. Some might view such uncertainty as a reason to avoid further investigation, but we would argue the opposite: High uncertainty brings with it high marginal value of information. Given the humanitarian stakes, this also suggests that even modest research efforts on GCBRs is of very high value.

Much of this research is already being done by the health security community. For example, the core capacities being built through the Global Health Security Agenda will be important regardless of whether a disease incident is small-scale bioterrorism, a natural epidemic, or a catastrophic outbreak that shapes future generations (and hopefully would prevent this from ever occurring). Building on this work and understanding how well it addresses GCBR or whether a specific research agenda will be needed seems like a natural next step.

One unresolved question for future research might be what we call the "anthropogenic GCBR hypothesis," which states that the vast majority of GCBR comes from human agency rather than natural sources. Nick Bostrom suggests this when he writes:

... the great bulk of existential risk in the foreseeable future consists of anthropogenic existential risks - that is, those arising from human activity. In particular, most of the biggest existential risks seem to be linked to potential future technological breakthroughs that may radically expand our ability to manipulate the external world or our own biology. As our powers expand, so will the scale of their potential consequencesintended and unintended, positive and negative. ${ }^{2(\mathrm{p} 16)}$

In the specific case of GCBRs, there are many theoretical arguments supporting the anthropogenic GCBR hypothesis, but most have yet to be thoroughly investigated. Some of these arguments for natural GCBR being unlikely might include:

- Humanity has survived hundreds of thousands of years without an extinction level GCBR, so a priori it is fairly unlikely that a GCBR that our ancestors faced would emerge in the next century or so. Research on extinction level GCBRs should therefore focus on novel factors that our ancestors did not face, such as air travel, factory farming, or biotechnology.

- The number of mammalian species that have gone extinct due to disease (as opposed to other factors) is very limited, with just 1 confirmed example. Cataloguing and analyzing "GCBR-like" events across a wide variety of species would be another good starting point for evaluating these risks.

- Optimal virulence theory predicts that pathogens suffer a trade-off between virulence and transmissibility. This would suggest that natural evolution would be unlikely to produce disease at maximum lethality and transmissibility. However, the debate around optimal virulence theory is still not resolved and constitutes another ripe area for GCBR research.

While some arguments for higher anthropogenic GCBR might include:

- Gain-of-function research, state-run bioweapons programs, and accidental discoveries such as the 2001 mousepox strain with $100 \%$ CFR; these all have provided proofs

(C) Piers Millett and Andrew Snyder-Beattie, 2017; Published by Mary Ann Liebert, Inc. This Open Access article is distributed under the terms of the Creative Commons Attribution Noncommercial License (http://creativecommons.org/licenses/by-nc/4.0/) which permits any noncommercial use, distribution, and reproduction in any medium, provided the original author(s) and the source are credited. 
of principle that humanity can produce worse-than-natural diseases.

- Dual-use technologies, such as DNA synthesis, have undermined many of the technical barriers to acquiring or using biological weapons over the past decade, as noted by the IAP:Global Network of Science Academies.

- Biological attacks could use multiple diseases, or multiple releases of the same disease, to achieve higher casualties.

One final point might be that the potential for biotechnology is huge, and, given that science and technology have produced many surprises in the past, there could be additional unknown risks. One hundred years ago, nuclear weaponry would not have been on the horizon - likewise, we should expect that in the next hundred years there will be dangerous biotechnological breakthroughs that we can hardly imagine now.

Some might argue for a precautionary approach-that innovation ought to be slowed or halted altogether. We would strongly disagree. Some technologies will be essential for countering these risks. Rather, we should accelerate those technologies that offer the best hope of countering future risks or of keeping us safe, even if we are uncertain as to what those risks are. Examples of such work might include: further investment in better ways to design, test, build, and distribute medical countermeasures; a greater focus on applied biosafety and biosecurity research to develop the tools and technologies to keep us safe and secure; and better integration of social sciences to help ensure the work is embedded in the broader social context.

Having a common understanding of what a GCBR is, what characteristics it might possess, and how it is related to other risks is valuable. It helps to further broaden how we think about biological risk —adding another element to our efforts. This is a well-worn path. Recent years have seen different origins for biological risk (natural, accidental, or deliberate) brought together into a spectrum, helping to break down silos between those working to prevent, respond to, and mitigate these risks. The One Health approach has broken down barriers between those working on public health and animal health issues. Global health security efforts work to erode geographic differences in health capacity by building core health capacities. The next step is to create a GCBR community that provides a space for overlapping interests between the health security communities (with a focus on more likely biorisks) and the global catastrophic risk communities (that focus on the highestconsequence events). Building on the experiences of bringing the health and security communities together, it is important to explore similarities and differences in terminology, worldview, and culture. Working together to better understand GCBR is an excellent start.

\section{References}

1. Schoch-Spana S, Cicero A, Adalja A, et al. Global catastrophic biological risks: toward a working definition. Health Secur 2017; 15(4).

2. Bostrom N. Existential risk prevention as global priority. Global Policy 2013;4(1):15-31.

Piers Millett, Senior Research Fellow Andrew Snyder-Beattie, Director of Research University of Oxford Future of Humanity Institute Suite 1, Littlegate House 16/17 St Ebbes Street Oxford OX1 1PT UK

Email: andrew.snyder-beattie@zoo.ox.ac.uk 